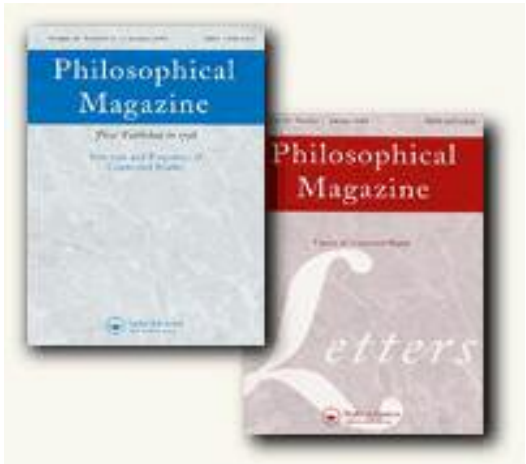

\title{
Controlling the plastic anisotropy in asymmetrically rolled aluminium sheets
}

\begin{tabular}{|r|l|}
\hline Journal: & Philosophical Magazine \& Philosophical Magazine Letters \\
\hline Manuscript ID: & TPHM-07-Nov-0346.R3 \\
\hline Journal Selection: & Philosophical Magazine \\
\hline $\begin{array}{r}\text { Date Submitted by the } \\
\text { Author: }\end{array}$ & $14-$ Mar-2008 \\
\hline Keyplete List of Authors: & $\begin{array}{l}\text { Sidor, Jurij; Materials Innovation Institute } \\
\text { Miroux, Alexis; Materials Innovation Institute } \\
\text { Petrov, Roumen; TU Delft } \\
\text { Kestens, Leo; TU Delft }\end{array}$ \\
\hline Keywords (user supplied): & aluminium alloys, deformation mechanisms \\
\hline & aluminium alloys, deformation mechanisms \\
\hline
\end{tabular}

\section{(5) ScholaronE" \\ Manuscript Central}




\title{
Controlling the plastic anisotropy in asymmetrically rolled aluminium sheets
}

\author{
J. SIDOR*1, A. MIROUX 1, R. PETROV 2,3, L. KESTENS 3
}

1 Materials Innovation Institute, Mekelweg 2, 2600 GA Delft, the Netherlands

2 Ghent University, Dept. of Metallurgy and Materials Science, Technologiepark 903, Ghent, Belgium

3 Delft University of Technologies, Dept 3ME, Mekelweg 2, 2600 GA Delft, the

Netherlands

*E-mail: j.sidor@tudelft.nl

Formability of metals depends on the crystallographic texture produced during thermo-mechanical processing. The crystallographic orientation of the deformed grains results from the applied deformation path. Asymmetric rolling is a new process which is expected to improve the formability of rolled aluminium sheets by introducing an intense shear deformation and the associated shear texture. The asymmetric rolling is applied to aluminium alloy AA6016 with roll diameters ratio of 1.5. Both full constraints and grain interaction models are employed to investigate the evolution of texture during conventional and asymmetric rolling processes. The superior planar and normal anisotropy values of asymmetrically rolled and annealed sheet over the conventionally produced one are interpreted in terms of differences in the deformed microstructures and the ensuing rolling and recrystallization textures.

Keywords: aluminum alloys, deformation mechanisms

\section{Introduction}

Use of light metals in automotive industry is an effective way for weight reduction of car bodies and thus for low fuel consumption with respective low emission of carbon dioxide. Aluminium alloys are attractive materials for automotive applications because of their favourable strength to weight ratio and high corrosion resistance. The major drawbacks to wide scale application of aluminium in the automotive industry are its comparatively high cost and its limited formability.

Generally, the formability is defined as the amount of deformation that can be given to a specimen without fracture or necking during a given process. Forming limit criteria for various processes depend on the ductility of the material under processing conditions. Thus, the formability of material is related to a continuous spectrum of deformation modes whereas the deep-drawing deserves particular attention because it is one of the most crucial elements in the complex strain paths associated with press forming of the car body panels. However, aluminium alloys have limited formability in comparison with steel sheets [1-3].

From the point of view of material's response during deformation, the formability of material is dependent on the work hardening coefficient, the strain-rate sensitivity and the normal anisotropy. The first two factors, which influence plastic instability and ductility, can be modified by adding alloying elements and appropriate heat treatment, 
whereas the normal anisotropy is closely related to the texture [1]. The normal anisotropy is characterized by $r$-value (Lankford value) which is defined as the ratio of the logarithmic strain $\varepsilon_{w}$ in the sample transverse direction to the logarithmic strain $\varepsilon_{h}$ in the sample normal direction during tensile test:

$$
r=\frac{\varepsilon_{w}}{\varepsilon_{h}}=\frac{\ln \left(w_{i} / w_{f}\right)}{\ln \left(h_{i} / h_{f}\right)}
$$

where $w_{i}, w_{f} h_{i}$ and $h_{f}$ are the widths and thicknesses before and after tensile deformation, respectively.

The $r$-value is calculated for a prescribed strain (e.g. 10\% elongation) in the direction of the tensile stress.

The variation of the $r$-value in the plane of the sheet (planar anisotropy) is conventionally characterised by the $\Delta r$ value. For this reason, the $r$-values are measured using specimens cut in three directions of $0^{\circ}, 45^{\circ}$ and $90^{\circ}$ with respect to the sheet rolling direction with respective $r_{0}, r_{45}$ and $r_{90}$ values. Hence, the average $\bar{r}$ and $\Delta r$ values are expressed:

$$
\begin{aligned}
& \bar{r}=\frac{\left(r_{0}+2 r_{45}+r_{90}\right)}{4} \\
& \Delta r=\frac{\left(r_{0}+r_{90}-2 r_{45}\right)}{2}
\end{aligned}
$$

Material with high $\bar{r}$ and low $\Delta r$ values is characterized by a low thickness reduction ratio during forming, which is beneficial for deep drawing applications.

In textured materials, such as commercial aluminium alloys, the Lankford value is highly anisotropic while its average measure still remains below 1 which is quite low compared to that of steel ( $\bar{r} \sim 2)$. The main reason of such large difference in $\bar{r}$ values between steel sheets with BCC structure and Al alloys with FCC structure is a specific texture formed during conventional rolling and subsequent annealing process. Both in BCC and FCC structured materials the $\gamma$-fibre texture (cf. Fig.1) is the favourable texture for obtaining an optimum deep-drawability. The $\{111\} / / \mathrm{ND}$ texture fibre naturally appears during the conventional processing of steel sheet while an entirely different type of crystallographic texture is obtained by conventional processing of aluminium sheet. It was shown that the texture of aluminium sheets produced by a conventional cold-rolling process is generally dominated by components that belong to the so-called $\beta$-fiber $[2,3]$. This texture is represented by a continuous set of orientations that runs from the Brass orientation $(\mathrm{B}-\{011\}<211>)$ through the $\mathrm{S}$ orientation $(\{123\}<634>)$ to the Copper orientation $(\mathrm{C}-\{112\}<111>)$. After annealing, the $\beta$-fiber texture often transforms to a cube-dominated texture $(\{100\}<001>)$, which is known as an unfavourable component for the formability of sheet metals and alloys. It is experimentally proven that a strong equivalence exists between shear texture in FCC material and rolling texture in BCC material and vice versa [4]. Accordingly, a shear texture in fcc metals features a strong intensity along the $\gamma$-fibre (as a rolling texture inbcc metals) along with other components." Among the various deformation processes that can produce a shear texture in aluminium alloys, the asymmetric rolling (ASR) is the easiest one to implement in an industrial scale and hence is of great interest for the 
producers. Asymmetric rolling can be achieved by imposing different circumferential speeds on the rolls in the rolling mill. This can be technically realized in two different ways (i) by changing the diameter of one of the rolls rotating with the same angular speed $[5,6]$ or (ii) by maintaining the same diameter for both rolls but varying the rotational velocity of one of them [7].

Many efforts have been made to obtain the favourable deep-drawability $\{111\} / / \mathrm{ND}$ texture in $\mathrm{Al}$ alloys by means of controlling the effective deformation mode during ASR process [5-10] but still there is a lack in understanding of the physical metallurgical aspects of this process. The current work aims to investigate the possibilities of the ASR process in $\mathrm{Al}$ alloys and to discuss them both by experimental (microstructure and texture measurements) and theoretical approaches (texture simulations).

\section{Experimental and Numerical Methods}

The material used in the current study is a hot rolled 6016 aluminium alloy. Microstructure and texture development were investigated during both conventional (CR) and asymmetric rolling (ASR) processes. The hot band strips with a thickness of $7.5 \mathrm{~mm}$ were first cold rolled to a final thickness of $4.5 \mathrm{~mm}$ and afterwards annealed at $360^{\circ} \mathrm{C}$ for $1 \mathrm{~h}$. Next, another cold rolling with a reduction of $73 \%$ followed by annealing at $360^{\circ} \mathrm{C}$ for $1 \mathrm{~h}$ was applied to the sheets. One group of the as processed sheets denominated as group A was subjected to cold asymmetric rolling (ASR) with $20 \%$ reduction and annealing at $550^{\circ} \mathrm{C}$ for $20 \mathrm{~s}$ and another group denominated as group B was subjected to cold symmetric rolling and annealing at $550^{\circ} \mathrm{C}$ for $20 \mathrm{~s}$.

For both groups A and B the asymmetric and symmetric rolling was applied in one pass and the final thickness of the sheets was $1 \mathrm{~mm}$. In the current ASR experiment, a rolling mill with rolls of different diameters rotating at the same angular speed was used. The ratio of the roll diameters applied in the present ASR process was 1.5.

The texture after cold rolling was determined by X-ray measurement of pole figures in the back reflection mode. From a set of four pole figures $\{111\},\{200\},\{220\},\{311\}$ the ODF was calculated using the conventional series expansion method [11]. The through thickness textures of the annealed specimens were measured by means of the EBSD technique. The EBSD system was attached to an FEI ${ }^{\circledR}$ Environmental Scanning Electron Microscope (ESEM XL30) with a $\mathrm{LaB}_{6}$ filament. The samples for EBSD examination were prepared by mechanical grinding and polishing according to the usual procedure with a final polishing step of $1 \mu \mathrm{m}$ diamond paste. The last preparation step was electrolytic polishing for $1 \mathrm{~min}$ at a voltage of $20 \mathrm{~V}$ with A2 Struers electrolyte cooled to a temperature of $-15^{\circ} \mathrm{C}$.

The EBSD measurements were carried out in the plane perpendicular to the sample transverse direction (TD-plane) crossing the entire thickness of the investigated sample. Two different scanning steps of $1 \mu \mathrm{m}$ and $2 \mu \mathrm{m}$ which varied in accordance to the grain size were employed in the present measurements. Results of the texture measurements are presented in the form of orientation distribution functions (ODF) in $\varphi_{2}=45^{\circ}$ const sections.

The gallium enhanced microscopy [12] has been applied for revealing the substructure of deformed materials. This technique is able to detect dislocation boundaries with misorientations below $1^{\circ}$.

The texture evolution as a function of different strain modes was predicted with the full constraints Taylor model (FCT) $[13,14]$ and two grain interaction models, the visco-plastic self consistent (VPSC) $[15,16]$ and the Alamel [17] models. The 
different crystal plasticity models predict the texture development with varying degrees of reliability due to different micro-mechanical approaches. The Taylor model assumes deformation uniformity within grains and across grain boundaries, violating the stress equilibrium condition at the interfaces. In reality, though, the strain of one grain is affected to some extent by its neighbours which is (partially) accounted for by the grain interaction models. In the Alamel model, both boundary conditions of strain compatibility and stress equilibrium are satisfied by consideration of inter-granular processes. This statistical (Monte-Carlo) model with a Taylor-type homogenisation requires two orders of magnitude less calculation than the crystal plasticity finite element method. The settings of the Alamel model used in the current investigation are the same as reported by Van Houtte et al [17]. In the visco-plastic self-consistent approach each grain is treated as an ellipsoidal visco-plastic inclusion embedded in an effective visco-plastic medium which is characterized by the average behaviour of all the grains. The deviation of the strain rate in the grain with respect to the macroscopic average one is characterised with the interaction parameter $n(n=10$ in the current VPSC simulations). The formal theory and a comprehensive derivation of the algebraic and tensorial equations of the visco plastic self-consistent formalism are presented by Tome and Lebensohn [18].

The initial experimentally measured texture was converted to a continuous ODF and subsequently discretized according to a method proposed by Toth and Van Houtte [19]. According to this procedure the total number of experimentally measured points is reduced to a set of 2000 equally weighted orientations. In the ODF calculations monoclinic sample symmetry was imposed for the ASR processed samples because shear deformation destroys the orthorhombic sample symmetry which is usually assumed for the conventional rolling process. In the texture simulations the orientation of each grain was updated after a deformation step of 0.05 . Only the $<111>\{110\}$ slip systems were taken into account.

The mentioned crystal plasticity models adopt the rate independent and rigid-plastic Voce-type law for work hardening [20] in which the evolution of the threshold stress $\tau^{s}$ with accumulated shear strain in each grain is expressed by:

$$
\tau^{s}=\tau_{0}^{s}+\left(\tau_{1}^{s}+\theta_{1}^{s} \Gamma\right)\left(1-\exp \left(-\Gamma\left|\theta_{0}^{s} / \tau_{1}^{s}\right|\right)\right)
$$

where $\Gamma$ is the accumulated shear in the grain; $\tau_{0}, \theta_{0}, \theta_{1}$ and $\left(\tau_{0}+\tau_{1}\right)$ are: the initial threshold stress, the initial hardening rate, the asymptotic hardening rate and the backextrapolated threshold stress, respectively.

In the current study, the Voce parameters were fitted to reproduce the experimental stress-strain curve. The following values were used in the simulation: $\tau_{0}=47.6 \mathrm{MPa}$, $\tau_{1}=49.5 \mathrm{MPa}, \theta_{0}=253 \mathrm{MPa}, \theta_{1}=11 \mathrm{MPa}$. A whole series of texture simulations was carried out with varying values of the material parameters but no significant differences in deformation textures were observed.

\section{Results and discussion}

\subsection{Initial microstructure}

Prior to the last cold rolling operation, the hot band was cold rolled with two intermediate annealing treatments at $360^{\circ} \mathrm{C}$ for 1 hour. The cold rolling deformation 
increases the sharpness of pre-existing $\beta$-fibre texture, whereas intensity of the cube orientation increases during subsequent annealing process. After the second intermediate annealing, the material displays a relatively homogeneous microstructure with elongated grains along RD, cf. Fig.2a. The deformation texture is not completely transformed to a typical recrystallization texture during the applied heat treatment. The through-thickness texture measurement of the annealed sheet (Fig.2b) reveals a strong cube component with an intensity of $\sim$ x 9 random levels and weaker transformation textures.

\subsection{Cold rolling}

Asymmetric rolling imposes shear deformation to the deformed sheets. Fig.3a shows the presence of sheared grains in the asymmetrically rolled material after a thickness reduction of $20 \%$. The directionality of the grain shearing is attributed both to the asymmetric rolling mode and high friction between rolls and the sheet. As revealed by the through-thickness texture measurement (cf. Fig.3b) the initial strong cube orientation (cf. Fig.2b) is completely destroyed and the texture featuring a $\gamma$-fibre components, as in shear textures, with local maximum intensity of $\sim \mathrm{x} 5$ random levels is developed after the unidirectional ASR process. Because of an inhomogeneous strain distribution across the thickness, different types of textures are observed through the thickness (cf. Fig.3 a-c). The top sub-surface, contacting the larger rolling cylinder, is characterized by strong shear orientations with local maxima at somewhat shifted E components (viz. Fig.1). Intensities of the $\gamma$ - fibre components in the midthickness region are two times higher than for the top sub-surface while the strength of $\mathrm{H}$ component (viz. Fig.1) is decreased. The strong shear texture within the midthickness layer of the sheet confirms the extension of the shear deformation zone into this region. The texture of the bottom sub-surface is represented by a weak $\theta$-fibre and somewhat displaced E orientations.

After the symmetric cold rolling with the same thickness reduction of $20 \%$, the shearing of the grains is less pronounced compared to the asymmetric rolling and also two direction of grain shearing could be observed on the IPF map. After this deformation process, the intensity of the pre-existing rolling texture increases (cf. Fig.4) while the strength of cube orientation does not decrease significantly which is related to the high symmetry of the exact cube orientation during compressive deformation.

\subsection{Strain distribution and texture modelling during cold rolling}

Crystal plasticity models such as the full constraints Taylor (FCT) model, the viscoplastic self-consistent model or the Alamel model can predict the texture evolution during plastic deformation. In the current study, the symmetric rolling process is approximated by plane-strain compression (PSC) deformation.

Generally, the similarity between the measured and predicted textures can be characterized by the texture index (TI) of the difference ODF $f_{1}(g)-f_{2}(g)$ :

$$
T I=\int\left[f_{1}(g)-f_{2}(g)\right]^{2} d g
$$

with $f_{1}(g)$ and $f_{2}(g)$ the ODFs of measured and calculated textures, respectively. Lower values of TI correspond to better prediction and in the case of identical textures $\mathrm{TI}=0$. 
The comparison between the experimentally measured texture (Fig.4b) and the predicted ones (Fig.5) by the FCT, the VPSC and the Alamel models shows a reasonable qualitative agreement. Among the applied crystal plasticity models, only the Alamel model produces the intensity of the cube orientation identical to the measured one.

As shown, the mentioned crystal plasticity models work quite satisfactorily for single strain mode operations such as rolling. In the case of the asymmetric rolling process, however, the strain displacement is more complex as compared to symmetric rolling. Generally, the deformation process of sheets under asymmetric rolling conditions can be approximated by a two-dimensional strain state of compressive strain along the normal direction plane $\left(e_{z z}\right.$ and $\left.e_{z z}=-e_{x x}\right)$ together with simple shear strain along the rolling direction $\left(e_{x z}\right)$. For this case, the resulting displacement gradient tensor is expressed as:

$$
\left[e_{i j}\right]=\left[\begin{array}{ccc}
e_{x x} & 0 & e_{x z} \\
0 & 0 & 0 \\
0 & 0 & e_{z z}
\end{array}\right]
$$

where the indices $x$ and $z$ indicate the rolling and normal directions respectively.

Both shear and compressive strain components imposed by the ASR process could be approximated by the calculations reported by S-B Kang et al. [21]:

$$
\begin{gathered}
\varepsilon_{z z}=\ln (1-\alpha) \\
\varepsilon_{x z}=\frac{2}{\left(h_{i}+h_{f}\right)}\left[R_{1} \cos ^{-1}\left(\frac{2 R_{1}-\alpha h_{i}}{2 R_{1}}\right)-R_{2} \cos ^{-1}\left(\frac{2 R_{2}-\alpha h_{i}}{2 R_{2}}\right)\right]
\end{gathered}
$$

where $\varepsilon_{i j}$ are the logarithmic strains, $h_{i}$ is the initial sheet thickness, $h_{f}$ is the final sheet thickness, $\alpha$ is thickness reduction, $R_{1}$ and $R_{2}$ are the roll radii.

Asymmetric rolling is a geometrically non-linear process where shearing of the elements is related to the difference in peripheral roll speeds. In this case, the equivalent strain cannot be calculated based on the linear integration of the infinitesimal strain tensor components as required by the conventional von Mises equation. An alternative equation obtained from literature [22, 23] is employed here to estimate the equivalent strain:

$$
\begin{gathered}
\varepsilon_{e q}=\frac{2}{\sqrt{3}} \phi \ln \frac{1}{1-\alpha} \\
\phi=\left[1+\left(\frac{(1-\alpha)^{2}}{\alpha(2-\alpha)} \tan \theta\right)^{2}\right]^{1 / 2}
\end{gathered}
$$

where $\theta$ is the apparent shear angle with respect to the normal direction. In the current asymmetric rolling experiment, the apparent angle of shearing, as estimated from the microstructural observations (cf. Fig.3a), is calculated to be $\theta \approx 47^{\circ}$. 
The deformation expressed by Eq.8 assumes a homogeneous strain mode across the thickness and disregards the contribution of redundant shear strain due to high friction between the sheet surface and the roll. The simulation with these simple assumptions allows us to investigate the physical phenomena involved in the processes. Assuming a constant strain velocity, the amount of shear imposed during the ASR process is characterized by the shear coefficient $K$ :

$$
K=\frac{\varepsilon_{x z}}{2 \varepsilon_{x x}}
$$

The shear strain approximation expressed by formula (8) clearly shows that development of shear deformation is strongly affected by the geometry of the rolls. Different roll diameter ratios account for various strain modes that have great impact on texture development. In order to investigate the crystallographic aspects of the asymmetric rolling process, the texture evolution is modelled with the VPSC model as a function of different combinations of plane strain compression and simple shear, which could be obtained in this rolling condition. Fig. 6 presents the results of the texture calculations obtained with a random distribution of orientations as the initial texture, imposing a constant von Mises deformation $\left(\varepsilon_{e q}=1.5\right)$. The predicted ODF in Fig.6a $(\phi=1)$ corresponds to the conventional rolling texture of FCC materials. As a consequence of the simple shear strain along the rolling direction $(\phi=1.8)$, the Copper component splits up into two orientations which move in opposite directions along the $<011>/ /$ TD fibre ( $\tau$-fibre) whereas the Brass component rotates towards the $\gamma$ - fibre $(\{111\} / / \mathrm{ND})$, cf. Fig.6b. The intensity of the newly created texture is significantly lower as compared to the case of symmetric rolling (i.e. pure plane strain compression). As shown in Fig.6c, a further increase of the shear ratio $(\phi=3.65)$ results in both the formation of an intense $\gamma$ - fibre and a rotated cube component $(\mathrm{H}$ orientation). Fig.6d presents the ODF section of the simulated deformation texture produced by simple shear only $(\phi \rightarrow \infty)$. It can be noticed that all the components rotate towards ideal shear deformation components consisting of a $45^{\circ}$ rotated cube orientation and the $\gamma$ - fibre with local maximum at $\{111\}<112>$ component (Fcomponent).

The presented calculations express the main features of texture development during cold rolling process with different amount of simple shear strain along the rolling direction.

In the current ASR process, the deformation is characterised by a high shear coefficient $(K \sim 1.8)$ that accounts for the development of shear textures. Fig.7 presents the results of texture modelling for the unidirectional ASR process by VPSC, Alamel and FC Taylor models assuming a homogeneous distribution of the deformation across the thickness. All calculations are in good qualitative agreement with EBSD texture measurements (viz. Figs. $3 \mathrm{~b}$ and 7 ). The calculated TI values reveal a better agreement between the Alamel model texture $(\mathrm{TI}=0.3)$ and the experimentally measured one ( $\mathrm{TI}=0.44$ for the FCT model and TI $=0.67$ for the VPSC model).

\subsection{Recrystallization texture and anisotropy}

Recrystallization in both asymmetrically and symmetrically rolled sheets accounts for various grain nucleation and growth mechanisms. After the asymmetric cold rolling process with $20 \%$ reduction and annealing at $550^{\circ} \mathrm{C}$ for $20 \mathrm{~s}$, the microstructure 
consists of a fine-grained homogeneous structure whereas the conventionally rolled and subsequently annealed material (cf. Fig.8) contains coarser and more elongated grains. The recrystallization textures are also different (Fig.9 a and b): after symmetric rolling the recrystallization texture is mainly a cube texture while the recrystallization texture of the asymmetrically rolled sheet is much weaker and displays maxima close to the L-component (cf. Fig.1), an intermediate component between $\mathrm{Cu}$ and cube and some scattered intensities close to the $\gamma$-fibre. This microstructural and textural variety can be attributed to distinctness in the applied strain modes.

The nucleation mechanism of grains could be related to the stored energy of the material. According to the Taylor theory of plastic deformation, the Taylor factor (TF) is a good measure of the dissipated plastic power and is used as measure of the stored energy in the material [24, 25] (assuming that the later is a fixed portion of the dissipated plastic power). The Taylor maps calculated by FCT model for both deformation modes are presented in Fig.9. The local minima in Taylor factor distribution for the asymmetric rolling process correspond to orientations close to the $\{111\} / /$ ND fibre. As shown in Fig.9d, a $45^{\circ}$ rotated cube orientation $\{001\}<110>$ and the Goss component $(\{110\}<001>)$ display the lowest Taylor factors in the case of plane strain compression deformation which characterises the conventional rolling process. The maxima in Taylor factor maps are close to the cube and a $90^{\circ}$ rotated Goss orientation (L-component), for ASR and PSC, respectively.

Another major difference between the two deformation paths is that in the asymmetric rolling the total imposed strain is considerably higher as compared to the former one. Substituting values $h_{x}, R_{x}, \alpha$ and $\theta$ into equations 9 and 10 gives an equivalent strain of $\varepsilon_{e q} \sim 0.55$ for $20 \%$ reduction in the ASR process whereas the equivalent strain at the same reduction level in conventional rolling is estimated to be $\varepsilon_{e q} \sim 0.26$.

In symmetrically rolled material with $20 \%$ reduction, a non-equilibrium grain boundary motion is induced by a small plastic deformation. The phenomenon in which a small prior plastic strain promotes the building of subgrains into adjacent smaller sub-grains of higher stored energy is widely known and interpreted as a strain induced grain boundary migration (SIBM) [25]. The growing recrystallized grains are the grains of the deformed structure displaying the lowest stored energy, i.e. a low TF. According to the deformed texture (Fig. 2) and the TF map (Fig. 9d) of the symmetrically rolled sheet, these grains are cube oriented grains, which is in accordance with the measured recrystallized texture (Fig.9b).

The same mechanism does not seem to apply during recrystallization after asymmetric rolling as the recrystallized texture does not correspond to the components of the deformed texture having minimum TF. It has been shown above that the equivalent strain for the asymmetric rolling process is $\sim 0.55$, which is higher than the usual range where SIBM is activated. The maxima of recrystallization texture are different from the main deformation texture components. This type of texture transformation is normally observed when the nucleation takes place on heterogeneities of the deformed microstructure. Indeed the deformed substructure obtained after asymmetric rolling presents clear shearing of the grains (cf. Figs.3a and 10). The finer recrystallized grain size and hence the higher density of nuclei obtained after ASR process as compared to conventional rolling can be related to this difference in nucleation mechanism resulting from the difference of strain path and amount of strain.

The texture development during recrystallization process affects both normal and planar anisotropy values. Fig.11 presents measured $r$-values in the investigated materials. As shown in Fig.9, the recrystallization texture in the asymmetrically rolled materials is considerably weaker than that in conventionally rolled material. 
Therefore, the asymmetrically rolled material exhibits the average r-value $(\bar{r}=0.92)$ which is comparable to randomly textured material $(\bar{r}=1)$. Furthermore, a somewhat shifted shear textures are still present in recrystallized material which significantly improves the r-value in the $45^{\circ}$ direction while conventionally rolled material shows an inverse tendency. Such an r-value profile could be beneficial to achieve a good formability in 6xxx alloys, especially in deep drawing applications.

\section{Conclusions}

Combination of plane strain compression and simple shear deformation results in rotation of rolling texture towards the shear texture components.

Both deformation and recrystallization textures developed in ASR process are considerably weaker in comparison to that obtained by conventional thermomechanical treatment.

The microstructure of asymmetrically rolled and subsequently annealed sheet reveals a fine-grained structure with an average grain size which is approximately three times smaller grain size as compared to conventionally processed material.

The presence of shear texture in recrystallized material significantly improves both planar and normal anisotropy values.

Generally, both the Taylor and the VPSC models give reasonable qualitative texture predictions for symmetric rolling with small thickness reduction but these models are less successful for the ASR process. The Alamel model is most successful of the ones employed in this study. It produces a very satisfactory agreement between measured and calculated textures both qualitatively and quantitatively.

\section{Acknowledgements}

This research was supported by M2i project (MC4.05231) in the framework of the Strategic Research program of the Materials Innovation Institute in the Netherlands. A special thanks to Prof. W.B. Hutchinson for valuable comments and discussions.

\section{References}

[1] H. Inoue, T. Yamasaki, G. Gottstein, P. Van Houtte, T. Takasugi, Materials Science Forum, 495-497, 573 (2005).

[2] I.L. Dillamore, W.T. Roberts, Acta Metallurgica, 12, 261 (1964).

[3] O. Engler, J. Hirsch, Mater Science and Engineering, A 336, 249 (2002).

[4] M. Holscher, D. Raabe, K. Lucke, Acta Metall. Mater, 42, 879 (1994).

[5] T. Sakai, K. Yoneda, Y. Saito, Materials Science Forum, 396-402, 309 (2002).

[6] K.H. Kim, D.N. Lee, Acta Mater, 49, 2583 (2001).

[7] T. Sakai, K. Yoneda, S. Osugi, Materials Science Forum, 495-497, 597 (2005).

[8] H. Jin, D.J. Lloyd, Materials Science and Engineering, A 465, 267 (2007).

[9] H. Inoue, M. Hori, T. Komatsubara, H. Tanaka, T. Takasugi, Materials Science Forum, 558-559, 207 (2007).

[10] T. Shimamura, T. Sakai, H. Utsunomiya, S. Kaneko, Materials Science Forum, 558-559, 1443 (2007).

[11] P. Van Houtte. MTM-FHM Software Version (second ed., MTM-KU Leuven, 1995), 65p.

[12] J. Hagstrom, O.V. Mishin, B. Hutchinson, Scripta Materialia, 49, 1035 (2003). 
[13] P. Van Houtte, E. Aernoudt, Zeitschrift für Metallkunde, 66, 202 (1975).

[14] P. Van Houtte, A.K. Kanjarla, A. Van Bael, M. Seefeldt, L. Delannay, European J. of Mechanics A/Solids, 25: 634 (2006);

[15] R.A. Lebensohn, C.N. Tomé, Acta Metall Mater, 41, 2611 (1993).

[16] U.F. Kocks, C.N. Tomé, H-R. Wenk, Texture and Anisotropy. Preferred Orientations in Polycrystals and their Effect on Materials Properties. (Cambridge University press, Cambridge, 1998), pp.466-509.

[17] P. Van Houtte, S.Y. Li, M. Seefeldt, L. Delannay, Int. J. of Plasticity, 21, 589 (2005).

[18] D. Raabe, F. Roters, F. Barlat, L-Q Chen. Continuum Scale Simulation of Engineering Materials: Fundamentals - Microstructures - Process Applications. (Wiley-VCH Verlag GmbH \& Co. KGaA, 2004), pp. 353-378.

[19] L.S. Toth, P. Van Houtte, Textures Microstruct. 19, 229 (1992).

[20] C.N. Tomé, G. R. Canova, U. F. Kocks, N. Christodoulou, J. J. Jonas, Acta metall. 32, 1637 (1984).

[21] S-B. Kang, B.K. Min, H.W. Kim, D.S. Wilkinson, J. Kang, Metall. and Mater. Transactions, A36, 3141 (2005).

[22] Q. Cui, C. Ohori, Materials Science and Technology. 16, 1095 (2000).

[23] Y. Saito, T. Sakai, F. Maeda, K. Kato, Tetsu-to-Hagane, 72, 799, (1986).

[24] L. Kestens, J.J. Jonas, Metall. and Mater. Transactions, A27, 155 (1996).

[25] F.J. Humphreys, M. Hatherly. Recrystallization and related annealing phenomena. (Pergamon, Oxford 2004), pp. 251-253. 


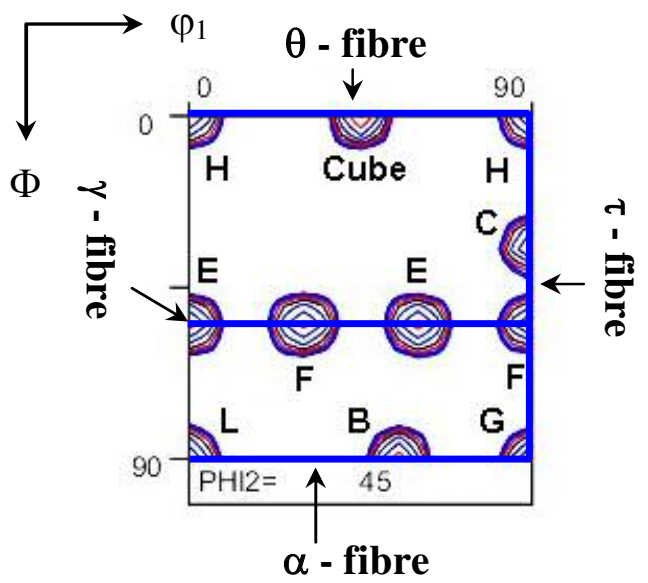

Fig.1. Main fibres and crystallographic orientations in reduced Euler space characterizing texture in $\mathrm{Al}$ alloys $\left(\varphi_{2}=45^{\circ} \mathrm{ODF}\right.$ section $)$.

(a)

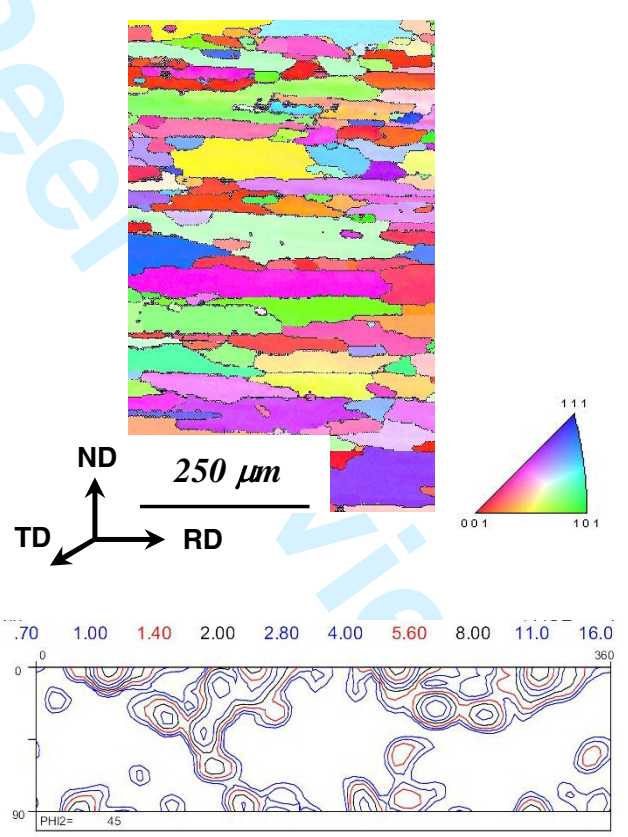

Fig.2. IPF map (a) and through-thickness texture (b) $\left(\varphi 2=45^{\circ} \mathrm{ODF}\right.$ section, the axes are the same as in Fig.1) of 6016 alloy prior to final cold rolling. 
(a)
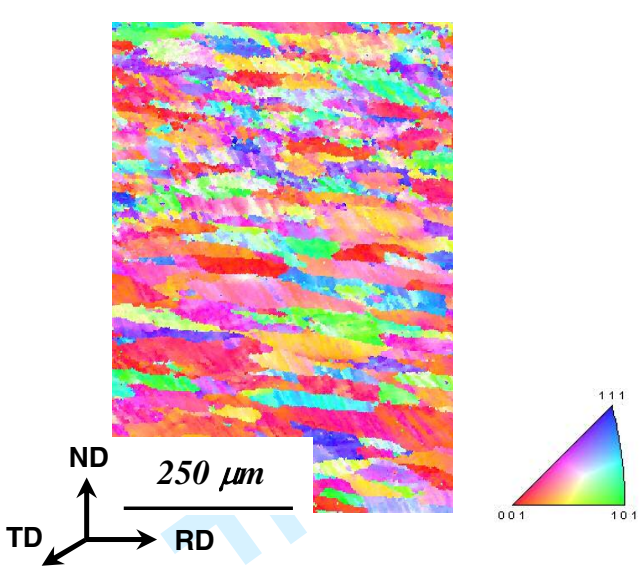

(b)

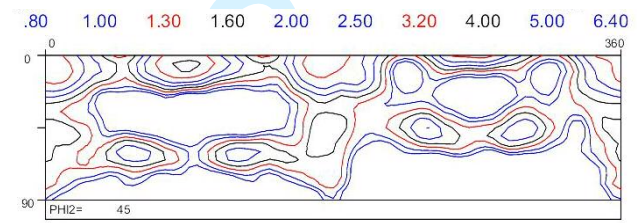

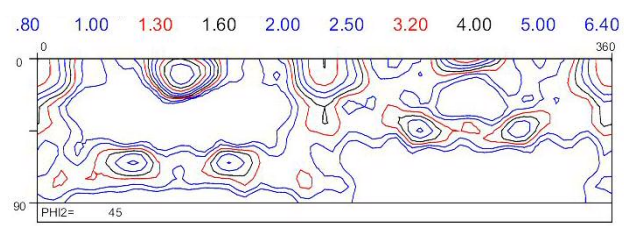
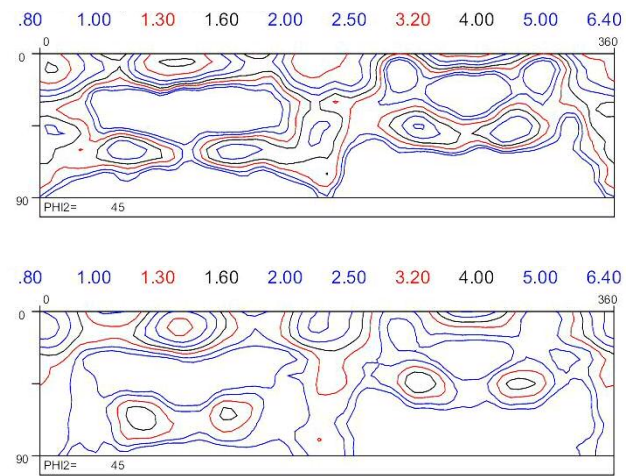

(c)

(d)

(e)

Fig.3. Microstructure and texture $\left(\varphi_{2}=45^{\circ} \mathrm{ODF}\right.$ sections $)$ development in asymmetrically rolled material A with 20\% thickness reduction: a) IPF map; b) a global throughthickness; texture; c) top sub-surface region contacting large roll; d) mid-thickness region; e) bottom sub-surface region contacting the small roll.

(a)

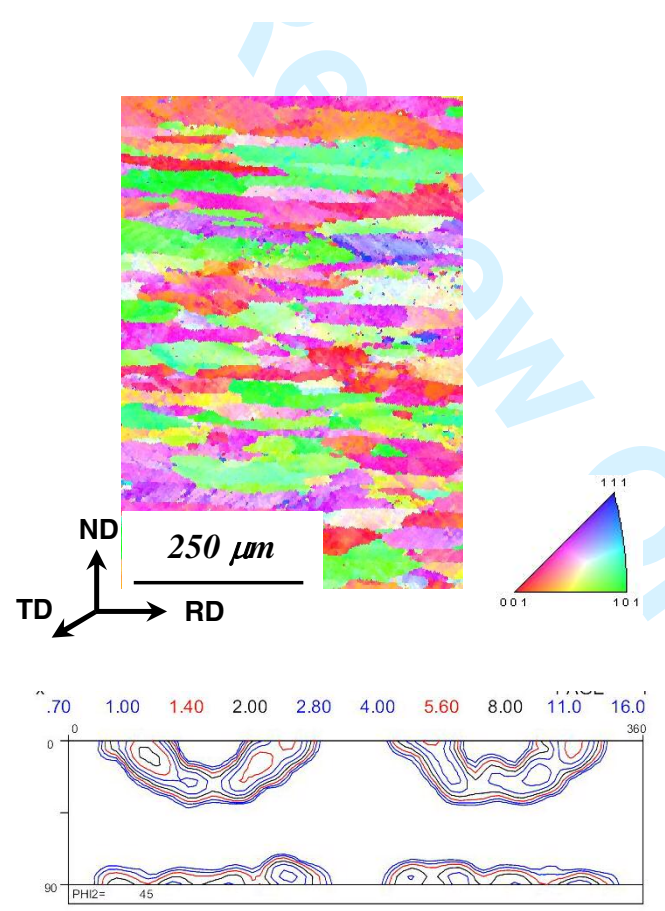

Fig.4. IPF map (a) and through-thickness texture (b) of symmetrically rolled material B with $20 \%$ thickness reduction. 
(a)

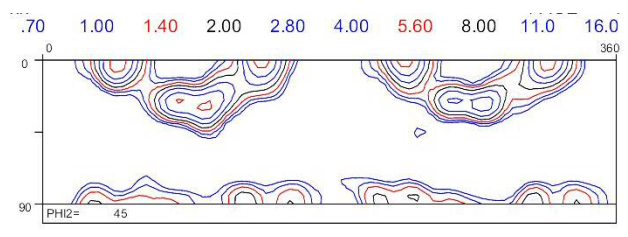

(b)

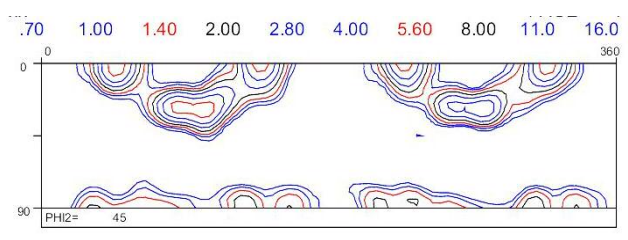

(c)

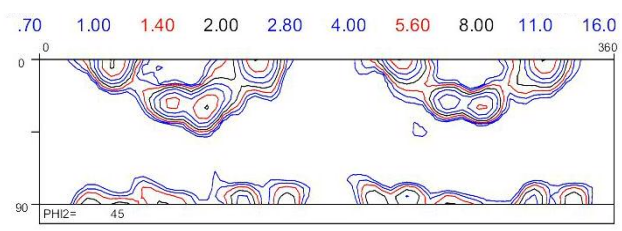

Fig.5. Predicted through-thickness texture for symmetric rolling process with initial texture of Fig.2b: a) Taylor model, TI=0.93; b) VPSC model, TI=0.94; c) Alamel model, $\mathrm{TI}=0.93$.

(a)

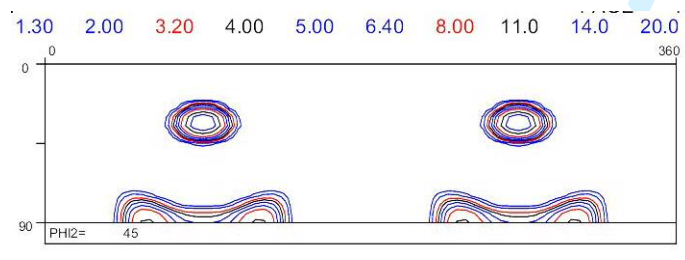

(b)

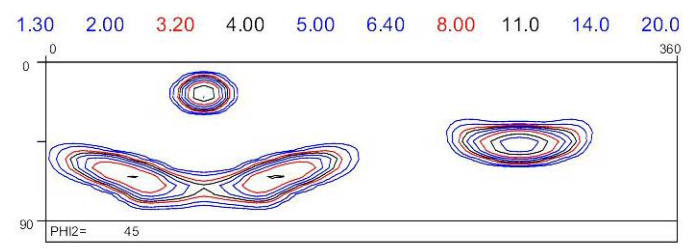

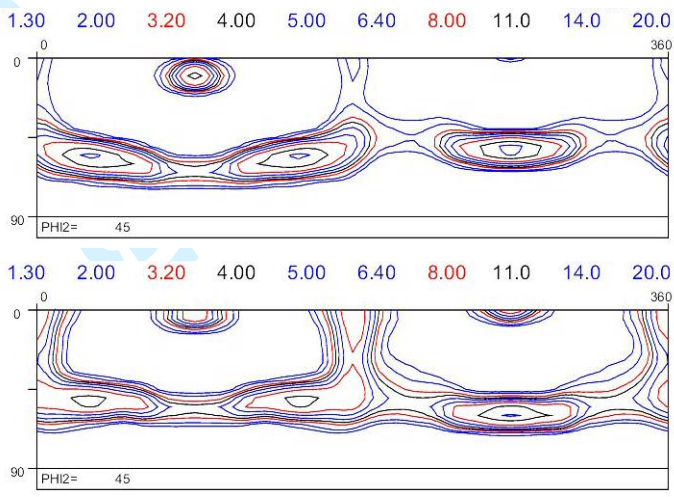

(c)

Fig.6. Results of texture simulation for various strain modes with the VPSC model at constant strain $\varepsilon_{e q}=1.5$ ( $\varphi_{2}=45^{\circ}$ ODF sections): a) $K=0, \phi=1, \alpha=0.725$; b) $K=1.5, \phi=1.82$, $\alpha=0.514$; c) $K=3.5$; $\phi=3.65, \alpha=0.3$ d) $K \rightarrow \infty, \phi \rightarrow \infty, \alpha \rightarrow 0$. Initial texture is random. 
(a)

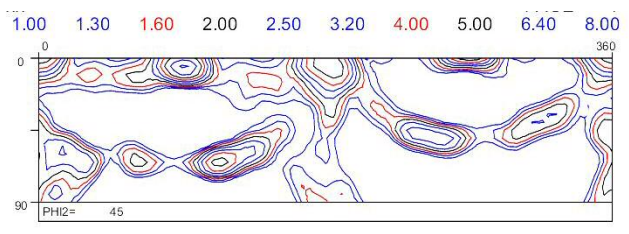

(b)

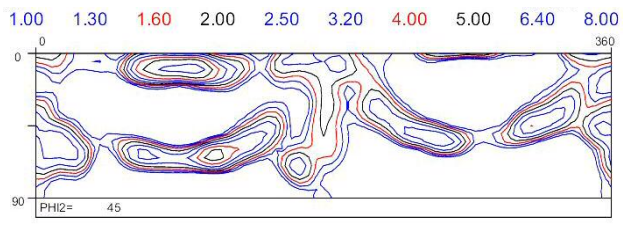

(c)

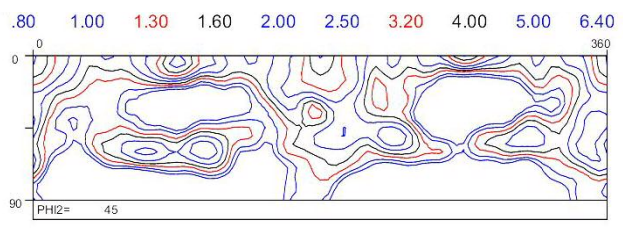

Fig.7. Predicted through-thickness texture for asymmetric rolling process with initial texture of Fig.2b: a) Taylor model, TI=0.44; b) VPSC model, TI=0.67; c) Alamel model, $\mathrm{TI}=0.3$.

(a)

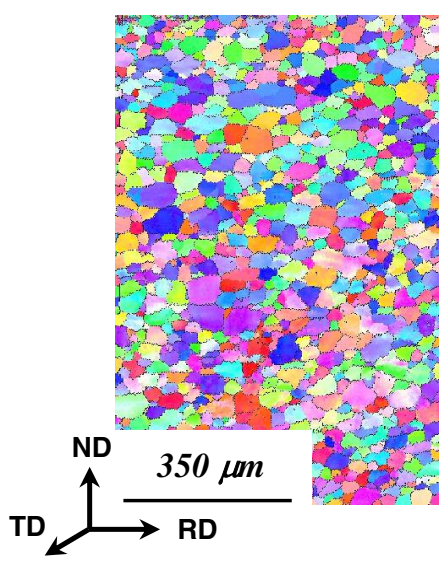

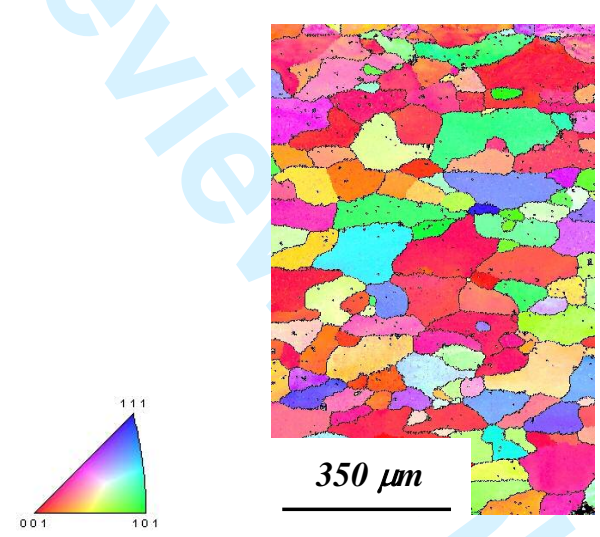

(b)

Fig.8. Microstructure of recrystallized sheets at $550^{\circ} \mathrm{C} / 20 \mathrm{~s}$ : a) asymmetrically rolled sheet A; b) symmetrically rolled sheet B. 
(a)

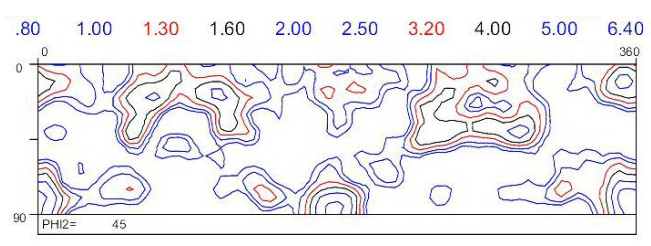

(c)

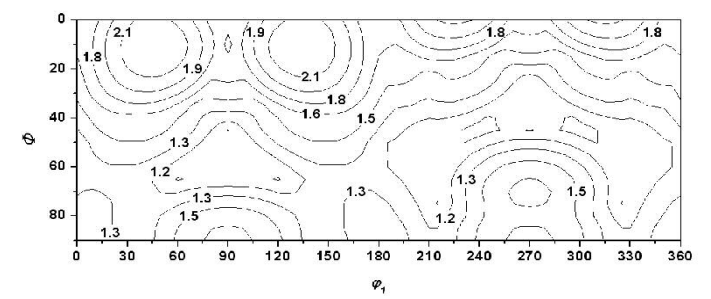

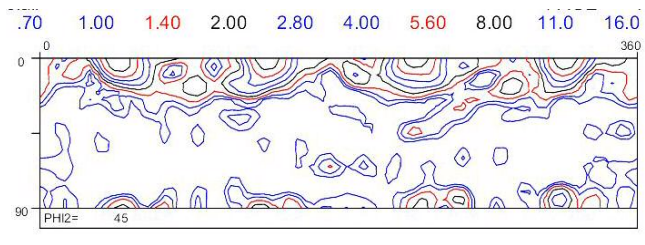

(b)

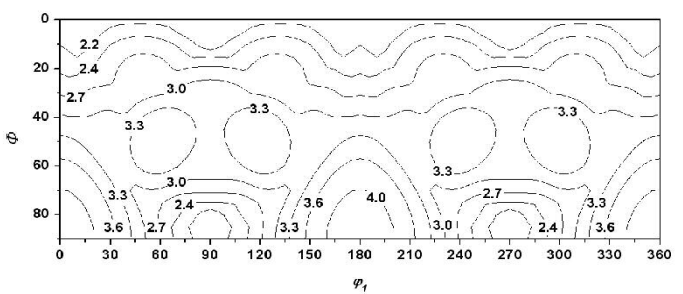

Fig.9. Recrystallization texture development and Taylor factor maps: a) asymmetrically rolled sheet A; b) symmetrically rolled sheet B; c) TF map for ASR process; d) TF map for symmetric rolling;
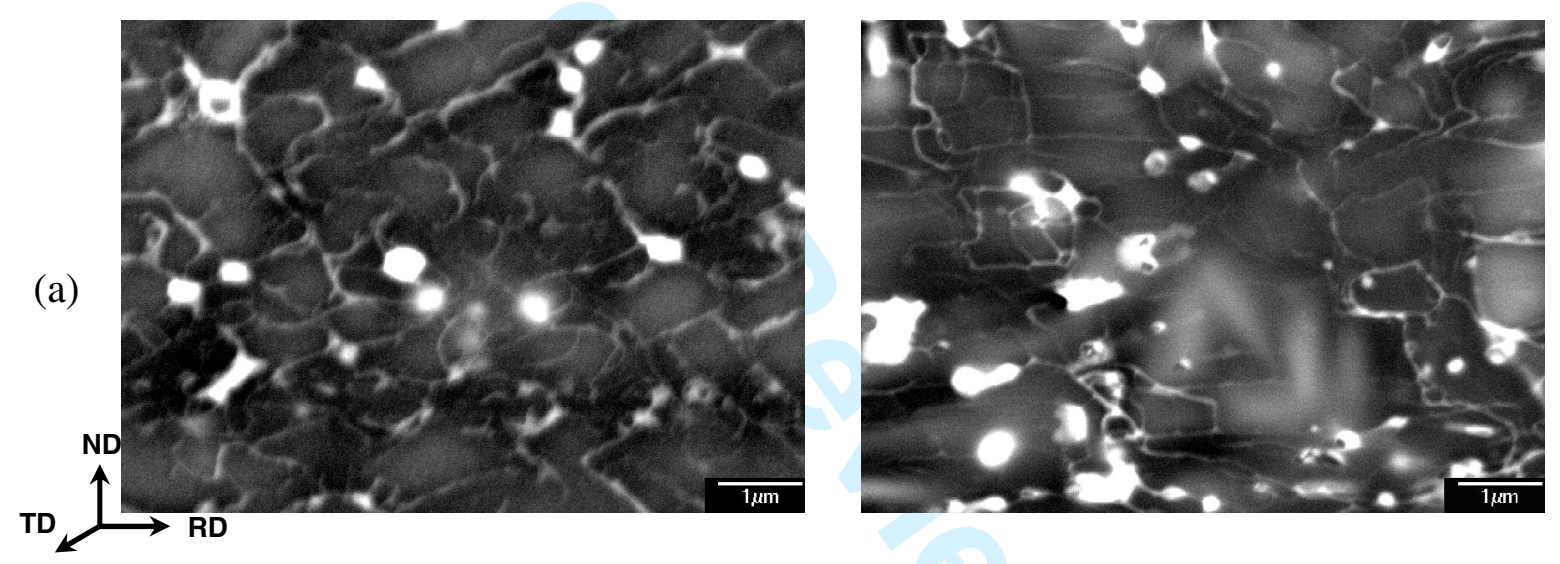

(b)

Fig.10. Substructure development in asymmetrically (a) and symmetrically (b) rolled materials with $20 \%$ thickness reduction.

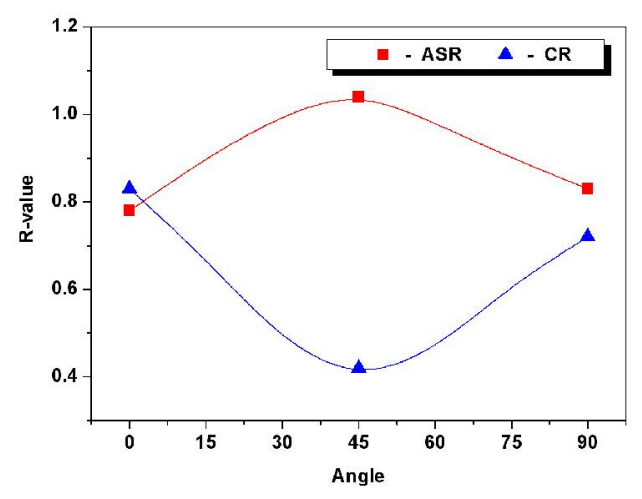

Fig.11. Measured r-values in asymmetrically (squares) and symmetrically (triangles) rolled materials after recrystallization process. 